\title{
Zeolitas Sódicas y Potásicas a partir de Cenizas Volantes Provenientes de la Combustión del Carbón de la Termoeléctrica Termotasajero S.A.S
}

\section{Sodic and Potassium Zeolites from Fly Ash Coming from Coal Combustion of Thermoelectric Termotasajero S.A.S}

\author{
Edgar Farid Carreño-Flórez ${ }^{\mathrm{a}^{*}}$ \\ Gabriel Peña-Rodríguez ${ }^{\mathrm{a}}$ \\ Martha Yasmid Ferrer Pacheco ${ }^{\mathrm{a}}$
}

Fecha de Recepción: 22.07.18

Fecha de Aceptación: 08.05.19

DOI: https://doi.org/10.19053/01217488.v10.n2.2019.8035

Resumen

Las Cenizas volantes (CV) de Termotasajero S.A.S, son un residuo de la combustión del carbón con alto impacto ambiental sobre el aire, el agua, el suelo y los ecosistemas. Poseen un alto contenido en Si y Al, lo que las convierte en un subproducto óptimo para la producción de zeolitas. Las CV se tamizaron y lixiviaron con el objeto de producir zeolitas de alta pureza. La síntesis se efectuó mediante el método de hidrogel en activación con álcalis de $\mathrm{NaOH}-2 \mathrm{M}$ y $\mathrm{KOH}-5 \mathrm{M}$, cristalización de $2 \mathrm{~h}$ y $3 \mathrm{~h}$ respectivamente, a $90{ }^{\circ} \mathrm{C}$ y 560 rpm. Las zeolitas sódicas y potásicas presentaron relación $\mathrm{Si} / \mathrm{Al}$ igual a 1,19 y 1,00 respectivamente, las cuales se clasificaron como zeolita natrosilita y zeolita K-F, de acuerdo al estudio por difracción de rayos $\mathrm{X}$ (DRX). En el producto zeolítico se identificaron que los elementos principales son $\mathrm{Si}, \mathrm{Al}, \mathrm{O}, \mathrm{Na}, \mathrm{K}$, $\mathrm{Ca}, \mathrm{Fe}$, y en proporciones menores $\mathrm{Ti}$ y $\mathrm{Mg}$, con morfología irregular de microcristales con tamaños de partículas inferiores a 12 micrómetros $(\mu \mathrm{m})$. El análisis de Capacidad de Intercambio Catiónico (CIC) deja en evidencia la alta capacidad de intercambio catiónico del material, encontrándose que el mayor valor (141 meq/100g) corresponde a las zeolitas activadas con $\mathrm{NaOH}$ y $(111,7 \mathrm{meq} / 100 \mathrm{~g})$ a las de $\mathrm{KOH}$.

Palabras clave: Cenizas Volantes, Método de Hidrogel, Zeolitas.

a Grupo de Investigación en Instrumentación y Física de la Materia Condensada (GIFIMAC), Facultad de Ciencias Básicas, Universidad Francisco de Paula Santander, Norte de Santander, Cúcuta, 540003, Colombia

* edgarfaridcf@ufps.edu.com

* gabrielpr@ufps.edu.com

* marthayasmidfp@ufps.edu.com 


\begin{abstract}
The fly ash (CV) of Termotasajero S.A.S, is a residue of coal combustion with high environmental impact on air, water, soil and ecosystems. They have a high content of Si and Al, which makes them an optimal by-product for the production of zeolites. The CV were sieved and leached in order to produce high purity zeolites. The synthesis was carried out by the hydrogel method in activation with alkalis of $\mathrm{NaOH}-2 \mathrm{M}$ and $\mathrm{KOH}-5 \mathrm{M}$, crystallization of $2 \mathrm{~h}$ and $3 \mathrm{~h}$ respectively, at $90^{\circ} \mathrm{C}$ and $560 \mathrm{rpm}$. The sodium and potassium zeolites presented a $\mathrm{Si} / \mathrm{Al}$ molar ration of 1.19 and 1.00 respectively, which were classified as zeolites natrosilite and zeolite $\mathrm{K}-\mathrm{F}$, according to the ray-X diffraction pattern (DRX). In the zeolitic product, the main elements were identified as $\mathrm{Si}, \mathrm{Al}, \mathrm{O}, \mathrm{Na}, \mathrm{K}, \mathrm{Ca}, \mathrm{Fe}$, and in smaller proportions $\mathrm{Ti}$ and $\mathrm{Mg}$, with irregular morphology of microcrystals with particle sizes less than 12 micrometers $(\mu \mathrm{m})$. The analysis of Cationic Exchange Capability (CIC) shows the ionic capability of the material, finding that the highest value $(141 \mathrm{meq} / 100 \mathrm{~g})$ corresponds to the zeolites activated with $\mathrm{NaOH}$.
\end{abstract}

Key words: Fly Ash, Hydrogel Method, Synthesis, Zeolites.

\section{INTRODUCCIÓN}

El carbón es el combustible primario de la central termoeléctrica de Termotasajero S.A.S, dependiendo de la disponibilidad de energía térmica y de la demanda general dentro del área de servicio se quema más o menos carbón anualmente. Teniendo en cuenta que por cada tonelada de carbón que se quema, se produce aproximadamente unos $220 \mathrm{~kg}$ de cenizas volantes (CV) estas originan problemas ambientales, debido a la acumulación de CV en grandes depósitos. El uso principal de las $\mathrm{CV}$ producidas por la central termoeléctrica, es para la producción de cemento, el cual le confiere propiedades de durabilidad y calidad al hormigón [1]. Las CV presentan composición química de acuerdo a la literatura [2], en mayor proporción óxidos de sílice $\left(\mathrm{SiO}_{2}\right)$, alúmina $\left(\mathrm{Al}_{2} \mathrm{O}_{3}\right)$, oxido férrico $\left(\mathrm{Fe}_{2} \mathrm{O}_{3}\right)$, oxido de calcio $(\mathrm{CaO})$, en menor proporción, generalmente al 5\%, óxido de magnesio $(\mathrm{MgO})$, sulfatos $\left(\mathrm{SO}_{4}{ }^{2-}\right.$ ), carbono elemental (C) y álcalis $\left(\mathrm{Na}_{2} \mathrm{O}\right.$ y $\left.\mathrm{KO}_{2}\right)$, $\mathrm{y}$ otros constituyentes en cantidades aún más reducidas, como compuestos de titanio, vanadio, manganeso, fósforo, germanio, galio, etc. Lo que convierte a las $\mathrm{CV}$ en un subproducto eficaz del cual se pueden obtener zeolitas de alto contenido de pureza [3]. Las zeolitas se definen [4] como aluminosilicatos cristalinos, microporosos, constituidas de un entramado tridimensional de aniones tetraédricos de $\left(\mathrm{SiO}_{4}\right)^{-4}$ y $\left(\mathrm{AlO}_{4}\right)^{-5}$, que se unen por compartición de átomos de oxígeno y moléculas de $\mathrm{H}_{2} \mathrm{O}$, los que son fácilmente intercambiables, en los poros huecos de la estructura, las diferentes coordinaciones de los tetraedros, así como la relación Silicio/Aluminio originan los diferentes tipos de zeolitas [5]. Dependiendo de las condiciones a las cuales se sometan las $\mathrm{CV}$ y el tratamiento que se aplique en el proceso de zeolitización se pueden obtener zeolitas de diferentes tipos con aplicaciones de gran interés a nivel industrial y ambiental. Este material por sus características físicoquímicas, tiene aplicaciones según González, et al., 2014, "en la conversión de los compuestos orgánicos en hidrocarburos, absorción de contaminantes presentes en el aire o el agua, la polimerización catalítica, mejorar el rendimiento en cultivos agrícolas", adsorción de $\mathrm{CO}_{2}$ a través del intercambio iónico y la formación de nanodepósitos en la estructura interna [7], las zeolitas también aumentan la retención de iones en el suelo generando una disminución en los niveles de contaminación de lixiviados producto de las actividades agropecuarias, con ello una menor contaminación de aguas subsuperficiales [8].

En la presente investigación con el objeto de producir zeolitas de alta pureza se realizó tamizado de las CV en tamiz No. 400 ( 38 $\mu \mathrm{m})$ y lixiviación en sistema abierto. Mediante tratamiento en hidrogel se sintetizaron zeolitas sódicas y potásicas a partir de $\mathrm{CV}$ en activación en álcalis con $\mathrm{NaOH}-2 \mathrm{M}$ y $\mathrm{KOH}-5 \mathrm{M}$. Las muestras se caracterizaron por Microscopia Electrónica de Barrido (MEB), con el que se identificó la morfológica de las zeolitas. Mediante Espectroscopía de Rayos X por Dispersión de Energía (EDS) se determinó la composición química de las zeolitas y a través de difracción de rayos $X(D R X)$ se realizó la identificación 
de la fase cristalina del material. Los análisis mencionados anteriormente tuvieron lugar en la Universidad Industrial de Santander (UIS), ubicados en la sede de Guatiguará. Finalmente se caracterizaron las zeolitas implementando análisis químico de Capacidad de Intercambio Catiónico (CIC) en el laboratorio de suelos agrícolas de la Universidad Francisco de Paula Santander (UFPS) con el fin de conocer el poder catalítico, y las posibles aplicaciones del material zeolítico.

\section{MATERIALES Y MÉTODOS}

\subsection{Síntesis de Zeolitas}

Las CV fueron obtenidas de la central termoeléctrica de Termotasajero S.A.S (Norte de Santander) las cuales fueron utilizadas en la síntesis de zeolitas. La central termoeléctrica suministro una cantidad de $5 \mathrm{~kg}$ de $\mathrm{CV}$, las cuales se manipularon de acuerdo a la norma colombiana [9]. Previo a la síntesis de zeolitas se realizó remoción de inquemados mediante tamizado manual durante 5 min en tamiz Gran Test ASTM No. $400(\sim 38 \mu \mathrm{m})$. Después se trataron las CV pasante tamiz No. 400 a través de lixiviación en sistema abierto, en el que se pesaron $50 \mathrm{~g}$ de $\mathrm{CV}$, se mezclaron en un erlenmeyer de $1000 \mathrm{ml}$ con 500 $\mathrm{ml}$ de agua destilada agitándose magnéticamente en rampa hotplate STIRRER model 181 durante un periodo de $300 \mathrm{~min}$, finalmente las muestras se sometieron al proceso de secado usando estufa de circulación forzada marca Memmert UF110 durante un período de $30 \mathrm{~min}$ a $60^{\circ} \mathrm{C}$.

La conversión de $\mathrm{CV}$ en zeolitas fue efectuada por medio de síntesis en hidrogel, que de acuerdo a lo declarado por Gómez, J. (2001): "es un proceso de cristalización que se desarrolla a partir de geles amorfos, el cual se despolimeriza y solubiliza liberando los compuestos de silicato y aluminato que deben reorganizarse para formar la estructura cristalina de la zeolita", mediante este método "el gel está compuesto por el aluminosilicato que resulta de la disolución de las CV con los álcalis" Murayama, 2002. La mezcla (CV-solución activante) se mantiene cierto tiempo en periodo de envejecimiento, en el que se diluye la sílice aumentando su concentración para reaccionar con el aluminio formando la estructura del aluminosilicato. Se mezcló $150 \mathrm{ml}$ de solución alcalina de $\mathrm{NaOH}$ y $\mathrm{KOH}$ respectivamente con
$50 \mathrm{~g}$ de CV lixiviada en erlenmeyer de $500 \mathrm{ml}$. La mezcla obtenida se calienta controladamente en sistema abierto de baño maría, vaso de precipitados de $1000 \mathrm{ml}$ (Fig. 1).

La síntesis de zeolitas comienza con la etapa de envejecimiento, en agitación magnética a $560 \mathrm{rpm}$, hasta alcanzar temperatura de fondo igual a $90^{\circ} \mathrm{C}$. Manteniendo constante la temperatura a $90^{\circ} \mathrm{C}$ se da comienzo al periodo de cristalización, durante $2 \mathrm{~h}$ para la mezcla en solución activante $\mathrm{NaOH}$ y $3 \mathrm{~h}$ la de $\mathrm{KOH}$. Posteriormente, se sometieron las muestras a tratamiento hidrotermico en autoclave ALL AMERICAN 75X a presión de 15 Psi, 120 ${ }^{\circ} \mathrm{C}$ y $30 \mathrm{~min}$. Se lavaron y filtraron las muestras usando papel filtro $(\sim 20 \mu \mathrm{m})$ marca Whatman con agua desionizada. Finalmente, se secó el material producido en estufa de circulación forzada marca Memmert UF110 durante 5h a $105{ }^{\circ} \mathrm{C}$. Las muestras se molieron en mortero y pistilo, rotularon y almacenaron en desecador para evitar el aumento de humedad.

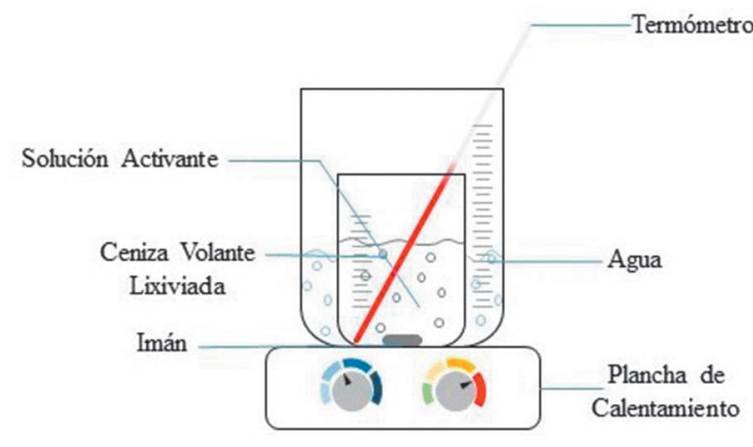

Figura 1. Diagrama baño maría.

\subsection{Caracterización de Zeolitas}

\subsubsection{Caracterización química por} espectroscopía de rayos $x$ por dispersión de energía (EDS)

La composición química de las zeolitas sintetizadas se identificó por medio del análisis de espectroscopía de rayos x por dispersión de energías (EDS), con el sensor de estado sólido del microscopio electrónico de barrido FEI Quanta FEG 650. El microanálisis por elemento químico usando (EDS) a cada una de las muestras de zeolitas sintetizadas, tanto a nivel superficial como puntual, se llevó a cabo con corrección ZAF ( $\mathrm{Z}=$ número atómico, $\mathrm{A}=$ absorción, $\mathrm{y}$ $\mathrm{F}=$ fluorescencia) a los espectros de energías 
de cada muestra. Para realizar dicho análisis se efectuó el proceso de metalización de la muestra en grafito, con el objeto de evitar que la muestra se cargue al ser excitada por los electrones, durante la generación del espectro (EDS).

\subsubsection{Caracterización Morfológica por} Microscopia Electrónica de Barrido (MEB)

Mediante microscopía electrónica de barrido (MEB), se conoció y describió la morfología superficial y la distribución granulométrica de las muestras sintetizadas, implementando software libre Image Tool ${ }^{\circledR}$ versión 3.0. El equipo usado para tal propósito fue el MEB FEI QUANTA FEG-650 (Fig. 2).

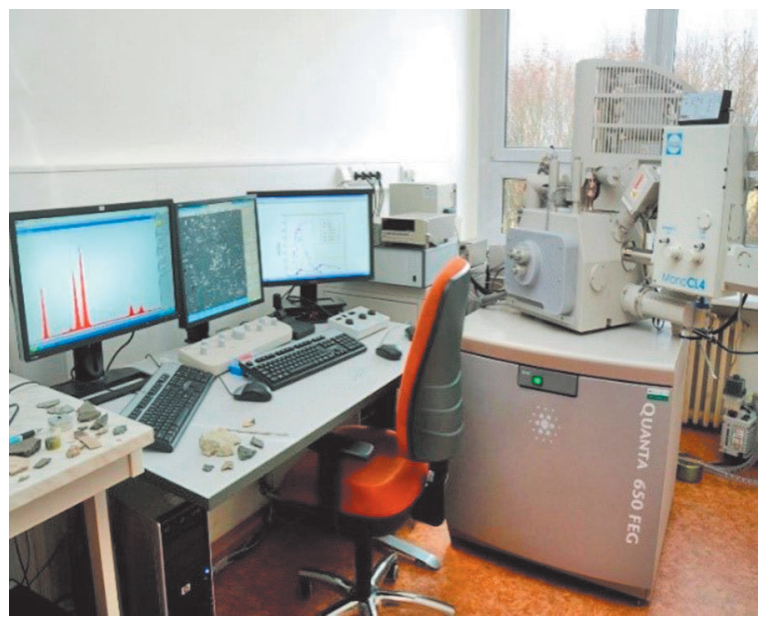

Figura. 2. Microscopio electrónico de barrido (MEB) FEI QUANTA FEG-650.

\subsubsection{Difracción de rayos $X(D R X)$}

La estructura cristalina de las muestras de zeolitas sintetizadas se determinó mediante difracción de rayos $\mathrm{X}$ (DRX), usando difractómetro BRUKER modelo D8 ADVANCE con geometría DaVinci bajo las siguientes condiciones: voltaje $(40 \mathrm{kv})$, corriente $(40 \mathrm{~mA})$, rendija de divergencia $(0,6 \mathrm{~mm})$, rendijas soller primario $\left(2,5^{\circ}\right)$, rendijas soller secundario $\left(2,5^{\circ}\right)$ tamaño de paso $\left(0,02035^{\circ} 2\right.$ Theta $)$, tiempo por paso $(0,6 \mathrm{~s})$, rango de registro $\left(3,5 \mathrm{a} 70^{\circ} 2 \mathrm{Theta}\right)$, radiación (CUKa1), filtro (níquel), uso de antidispersor de aire, detector lineal LynxEye, y barrido a pasos.

2.2.3. Capacidad de intercambio catiónico (CIC) La prueba de capacidad de intercambio catiónico (CIC), se efectuó en titulación con $\mathrm{NaOH} 0,1$ $\mathrm{N}$, con el objeto de evaluar la CIC del material sintetizado. El cálculo de CIC se determinó de acuerdo a la Ec. 1 [10]:

$C I C=\frac{P(\mathrm{Vm}-\mathrm{Vb}) * N N a O H *(100+\mathrm{pW})}{\mathrm{Wm}}($ Ec. 1$)$

En donde Vm es el volumen en mililitros de $\mathrm{NaOH}$ gastado en la titulación de la muestra, $\mathrm{Vb}$ es el volumen en mililitros de $\mathrm{NaOH}$ gastado en la titulación del blanco. $\mathrm{N}_{\mathrm{NaOH}}$ es la normalidad de $\mathrm{NaOH}, \mathrm{Wm}$ es el Peso en gramos de la muestra. $\mathrm{pW}$ es la humedad de la muestra a $105{ }^{\circ} \mathrm{C}$, expresada sobre base seca.

\section{RESULTADOS Y DISCUSIÓN}

\subsection{Tratamientos Previos a la Síntesis}

Una vez obtenida la ceniza volante, se procedió a determinar su granulometría usando la norma ASTM: C117-03, para lo cual se pesó una muestra de $2100 \mathrm{~g}$, separándose en 21 muestra de $100 \mathrm{~g}$, el cual fue pasado por la serie de tamices ASTM No. 325, 400, 500.

En la tabla 1, se presenta el resultado de la granulometría de la muestra. En esta se aprecia, que aproximadamente el 37,52\% de la muestra posee un tamaño de partícula mayor a $45 \mu \mathrm{m}$, mientras que el 28,60\% se encuentra en el rango entre 45 y $38 \mu \mathrm{m}$, y el $32 \%$ está entre 38 y $25 \mu \mathrm{m}$, así mismo, sólo el 1,88 \% de la muestra presenta un tamaño inferior a $25 \mu \mathrm{m}$. Por otra parte, se observa que el $62,48 \%$ de la muestra presenta una distribución de tamaño de partícula pasante tamiz No. $325(\sim 45 \mu \mathrm{m})$. 
Tabla 1. Granulometría de la ceniza volante de Termotasajero.

\begin{tabular}{|c|c|c|c|c|}
\hline \multicolumn{2}{|c|}{ Tamiz } & \multirow{2}{*}{$\begin{array}{c}\text { Peso Retenido } \\
\text { (gr) }\end{array}$} & \multirow{2}{*}{$\begin{array}{c}\text { Mat. Retenido } \\
(\%)\end{array}$} & \multirow{2}{*}{$\begin{array}{c}\text { Mat. Pasante } \\
(\%)\end{array}$} \\
\hline No. & $(\mu \mathrm{m})$ & & & \\
\hline 325 & 45 & 787,95 & 37,52 & 62,48 \\
\hline 400 & 38 & 600,58 & 28,60 & 33,88 \\
\hline 500 & 25 & 672,18 & 32,00 & 1,88 \\
\hline \multirow{3}{*}{\multicolumn{2}{|c|}{$\begin{array}{l}\text { Fondo } \\
\text { Perdidas } \\
\text { Total }\end{array}$}} & 18,87 & 0,90 & 0 \\
\hline & & 0,98 & 0 & \\
\hline & & 100 & 0 & \\
\hline
\end{tabular}

La granulometría de las CV de Termotasajero S.A.S poseen granulometría con tamaños de partícula inferiores a $250 \mu \mathrm{m}$, ya que solo el $62.48 \%$ de la muestra posee tamaños de partículas inferiores a $45 \mu \mathrm{m}$, hecho que se confirma con lo reportado por Peña y Ortega, 2014., al ser comparados con los resultados de la tabla 1.

En la (Fig. 3), se presenta una imagen digital de las muestras de CV correspondiente al pasante tamiz No. 325 y 400. De ésta, se observa la variación del cambio de color de gris oscuro (ver Fig. 3 a)) a gris claro (ver Fig. 3 b)), lo anterior se debe a la presencia de partículas de carbón inquemado, hecho que fue también reportado por [1].

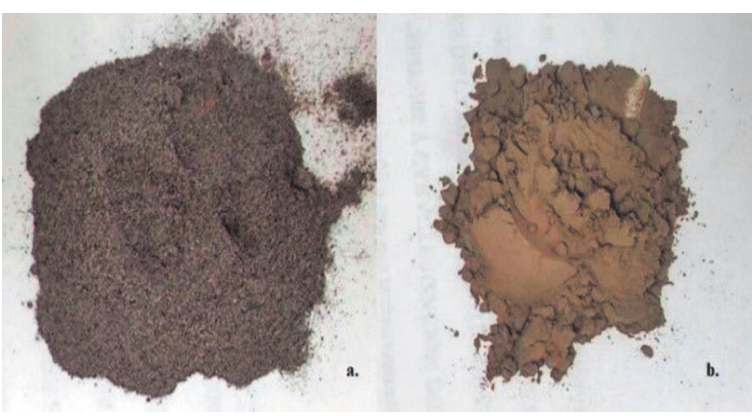

Figura. 3. Ceniza volante tamizada. a) Pasante No. 325, b) Pasante No. 400. Fuente.

Las técnicas de separación por tamizado permiten separar el carbón inquemado de las cenizas volantes producidas en los procesos de combustión de carbón, mejorando la calidad de las cenizas, permitiendo aplicaciones tecnológicas de éstas [12]. Por lo anterior, a fin de lograr cenizas con un porcentaje elevado de pureza, se identificó que la muestra pasante tamiz ASTM No. 400, son óptimas para la posterior etapa del proceso de síntesis de zeolitas, tal y como lo reporta Saavedra, et al., 2015.

La composición química mayoritaria en peso $(\% \mathrm{p} / \mathrm{p})$ de metales en las partículas ceniza volante de Termotasajero S.A.S, es el 38,56\% hierro $(\mathrm{Fe})$, en menor concentración $0,66 \%$ cobre $(\mathrm{Cu})$, $1,45 \%$ titanio (Ti), de acuerdo a lo reportado por Peña y Ortega. 2014.

Lo anterior justifica la realización del tratamiento previo de lixiviación con el objeto de reducir o eliminar el contenido de elementos metálicos pesados. Se evidencia la separación de los elementos metálicos ( $\mathrm{Fe}, \mathrm{Cu}, \mathrm{Ti})$ de las $\mathrm{CV}$ los cuales están relacionados según el color oscuro característico del hierro $(\mathrm{Fe})$ en mayor concentración de acuerdo a lo declarado por CEDEX, (2011). El material retirado de las muestras se presenta en la figura $4 \mathrm{~b}$ ), el cual es recaudado y depositado en recipientes de recolección.

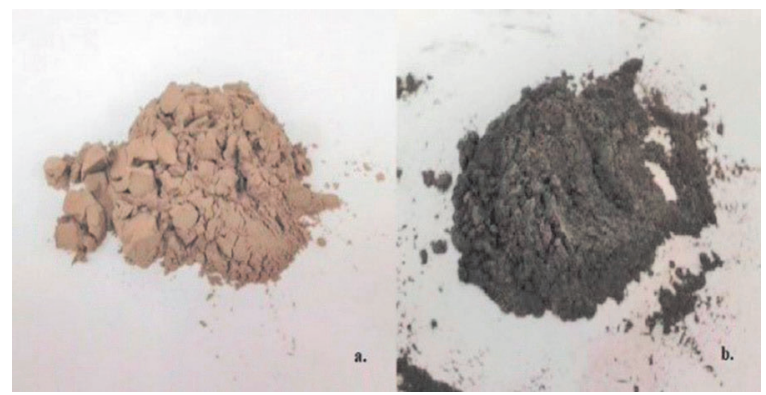

Figura. 4. a) CV lixiviada libre de humedad, b) Material extraído por lixiviación. 


\subsection{Caracterización del Producto Zeolítico por EDS, MEB, DRX y CIC}

En la tabla 2, se presentan los resultados del microanálisis para la superficie de las muestras sintetizadas de zeolitas sódicas (ZEONa) y zeolitas potásicas (ZEOK) (ver Fig. 5 y 6). El carbón presente en el microanálisis, es debido al proceso de metalización de la muestra.
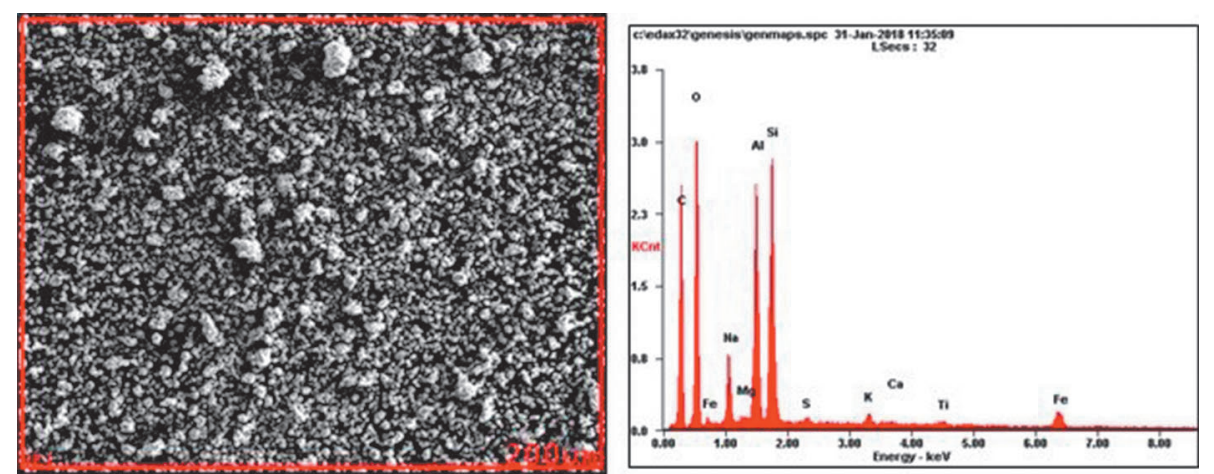

Figura. 5. Imágenes del espectro de dispersión de energía de rayos x, de las ZEONa marcadas en un área de $200 \mu \mathrm{m}$

Tabla 2. Resultados del microanálisis por elemento químico de las muestras ZEONa y ZEOK. Analizadas para el área resaltada en la figura 5 y 6.

\begin{tabular}{ccc}
\hline Elemento & ZEONa $(\% \mathbf{W t})$ & ZEOK $(\% \mathbf{\% t})$ \\
\hline $\mathrm{C}$ & $38,93 \pm 1,40$ & $27,83 \pm 1,58$ \\
$\mathrm{O}$ & $32,08 \pm 0,33$ & $32,45 \pm 0,50$ \\
$\mathrm{Na}$ & $3,79 \pm 0,12$ & $0,37 \pm 0,10$ \\
$\mathrm{Mg}$ & $0,20 \pm 0,06$ & $0,29 \pm 0,10$ \\
$\mathrm{Al}$ & $9,43 \pm 0,31$ & $12,99 \pm 0,81$ \\
$\mathrm{Si}$ & $11,17 \pm 0,43$ & $12,74 \pm 0,21$ \\
$\mathrm{~S}$ & $0,25 \pm 0,01$ & $0,23 \pm 0,02$ \\
$\mathrm{~K}$ & $0,58 \pm 0,06$ & $8,31 \pm 0,22$ \\
$\mathrm{Ca}$ & $0,25 \pm 0,01$ & - \\
$\mathrm{Ti}$ & $0,49 \pm 0,02$ & $0,57 \pm 0,03$ \\
$\mathrm{Fe}$ & $2,86 \pm 0,12$ & $4,25 \pm 0,03$ \\
$\mathrm{Relación} \mathrm{Si/Al}$ & 1,19 & $\sim 1,0$ \\
\hline
\end{tabular}



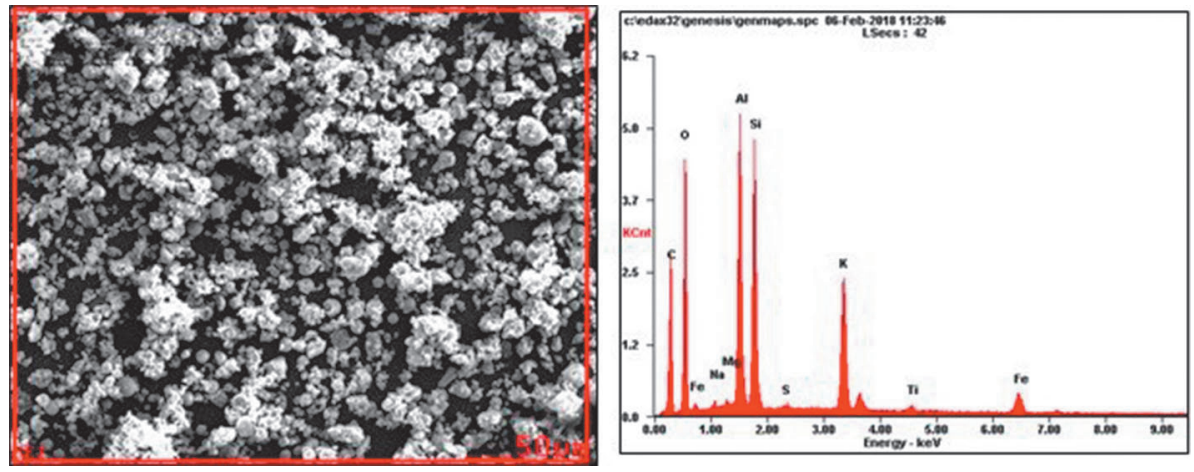

Figura. 6. Imágenes del espectro de dispersión de energía de rayos x, de la muestra ZEOK marcadas en un área de $50 \mu \mathrm{m}$

En las figuras 7 y 8 se observa el microanálisis puntual realizado a las muestras de zeolita sintetizadas (ZEONa y ZEOK) respectivamente, donde se aprecia una marca $(+)$ de la partícula a la cual se le realizó el análisis cuantitativo de la composición química, junto con su respectivo espectro.

Se evidencia en la tabla 3 y 4 la cuantificación en porcentaje en peso $(\% \mathrm{p} / \mathrm{p})$ de los elementos encontrados en la muestra de ZEONa y ZEOK del análisis puntual, en donde se aprecia que el pico de mayor intensidad es el correspondiente al silicio $(\mathrm{Si})$ y aluminio $(\mathrm{Al})$, característicos de las ZEONa, como previamente lo reporta Jiménez, (2004)., y los valores por elemento químico con mayor \%p/p propio de las ZEOK son $(\mathrm{K}, \mathrm{Si}, \mathrm{Al})$ [17]. Por otra parte, los contenidos de sodio son inferiores al $5 \%$ en las ZEONa y los de potasio en ZEOK al 28\%.

Las impurezas (S, Mg y Ti) que se pueden observar sobre las marcas por área y puntuales del estudio del EDS actuaron como una fase no reactiva en la síntesis de las zeolitas como lo reportan Benavides \& Mendoza, (2007).
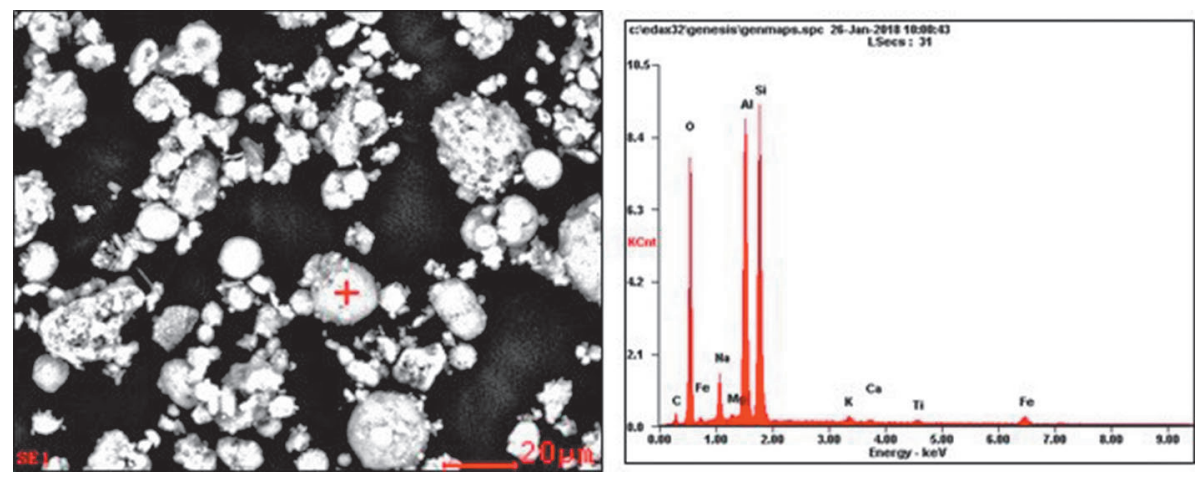

Figura. 7. Imágenes del espectro de dispersión de energía de rayos x de las ZEONa (análisis puntual).
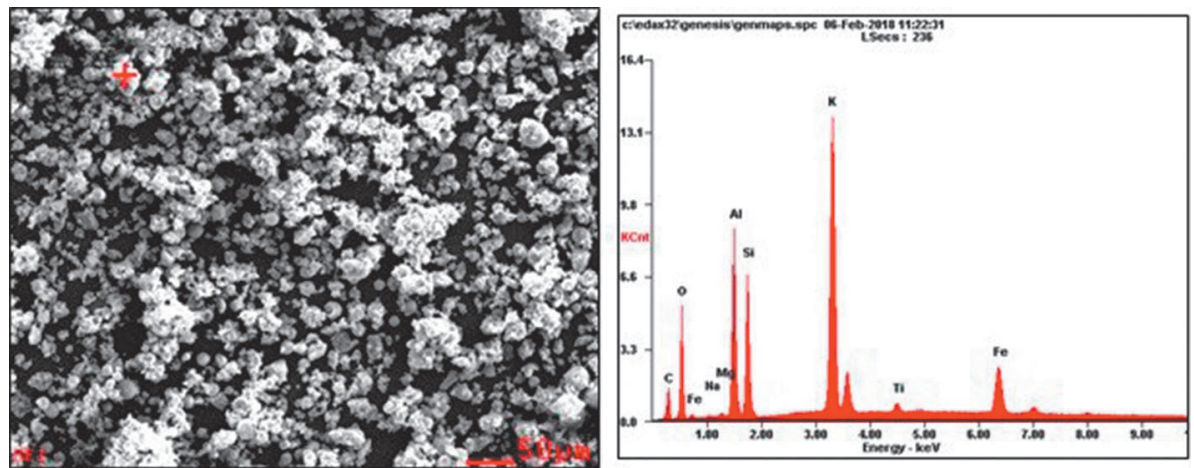

Figura. 8. Imágenes del espectro de dispersión de energía (EDS) de la muestra ZEOK (análisis puntual). 
Estas zeolitas (ZEONa y ZEOK) se consideran de baja relación $\mathrm{Si} / \mathrm{Al}$ [19] porque están entre $1 \leq \mathrm{Si} / \mathrm{Al}<5$. El bajo contenido de sílice de las zeolitas le confiere la característica de un alto poder hidrófilo y puede fijar o intercambiar iones más hidratados [20].

Tabla 3. Resultados microanálisis por elemento químico puntual de la muestra ZEONa. (figura 8)

\begin{tabular}{cc|c}
\hline Elemento & ZEONa $(\% \mathrm{Wt})$ & Relación Si/Al \\
\hline $\mathrm{C}$ & 5,19 & \\
$\mathrm{O}$ & 38,39 & \\
$\mathrm{Na}$ & 4,36 & \\
$\mathrm{Mg}$ & 0,34 & \\
$\mathrm{Al}$ & 20,53 & 1,33 \\
$\mathrm{Si}$ & 27,30 & \\
$\mathrm{~K}$ & 0,62 & \\
$\mathrm{Ca}$ & 0,31 & \\
$\mathrm{Ti}$ & 0,56 & \\
$\mathrm{Fe}$ & 2,49 & \\
\hline
\end{tabular}

Los resultados de la relación $\mathrm{Si} / \mathrm{Al}$ de las ZEONa al activar las $\mathrm{CV}$ con $\mathrm{NaOH}$, son similares a los reportados en [21], [22], [23], correspondientes a zeolitas-Na entre $\mathrm{Si} / \mathrm{Al}$ 1-1,6. La baja relación $\mathrm{Si} / \mathrm{Al}$ de las ZEOK se justifica por lo mencionado por Flores, et al., 2017, ya que la presencia de un mayor \%p/p de Fe causa una disminución en la cristalización de las zeolitas y hace que sea difícil disolver el silicio y el alumino en el medio de reacción, provocando una caída en la relación $\mathrm{Si} / \mathrm{Al}$. Esto ocurre debido a que los óxidos de hierro tienden a ocupar la superficie de las partículas $\mathrm{CV}$.

Tabla 4. Resultados espectro de rayos x (EDS) puntual de las ZEOK. Análisis de concentraciones por elemento químico.

\begin{tabular}{cc|c}
\hline Elemento & ZEOK $(\% \mathrm{Wt})$ & Relación Si/Al \\
\hline $\mathrm{C}$ & 8,67 & \\
$\mathrm{O}$ & 23,14 & \\
$\mathrm{Na}$ & 0,2 & \\
$\mathrm{Mg}$ & 0,26 & \\
$\mathrm{Al}$ & 12,59 & \\
$\mathrm{Si}$ & 11,28 & \\
$\mathrm{~K}$ & 27,69 & $\sim 1,0$ \\
$\mathrm{Ti}$ & 1,45 & \\
$\mathrm{Fe}$ & 14,74 & \\
\hline
\end{tabular}

En la figura 9, se observa la microfotografía de la muestra de ZEONa, realizada con una magnificación de 2000X. Los diámetros promedios de las partículas de estudio son de 7,57 $\mu \mathrm{m}$, con una desviación estándar de 0,75 , valor mínimo de $6,07 \mu \mathrm{m}$, y un valor máximo de $9,02 \mu \mathrm{m}$.

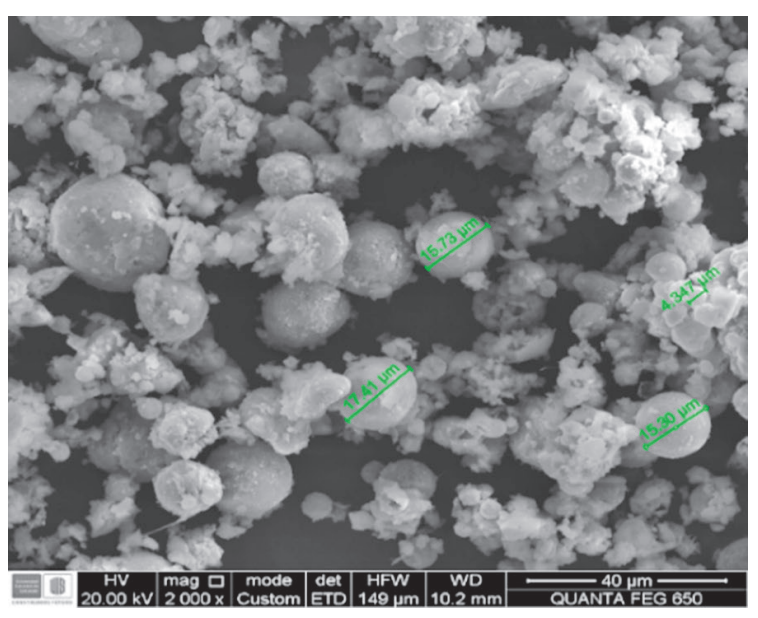

Figura. 9. Microfotografías de la muestra ZEONa, usando MEB FEI Quanta FEG 650.

Se registra el tamaño medio de las semiesferas A, B, C y D que corresponden a 11,66 $\mu \mathrm{m}, 4,94 \mu \mathrm{m}$, $7,96 \mu \mathrm{m}$ y $6,05 \mu \mathrm{m}$ respectivamente (ver Fig. 10).

La distribución de diámetros de partícula de las ZEONa presenta una magnitud comprendida entre $4,94 \mu \mathrm{m}$ y $11,66 \mu \mathrm{m}$, lo que confirma que las zeolitas tienen tamaños de partícula alrededor de 1-100 $\mu \mathrm{m}$ tal y como lo reporta Mosqueda, (1997).

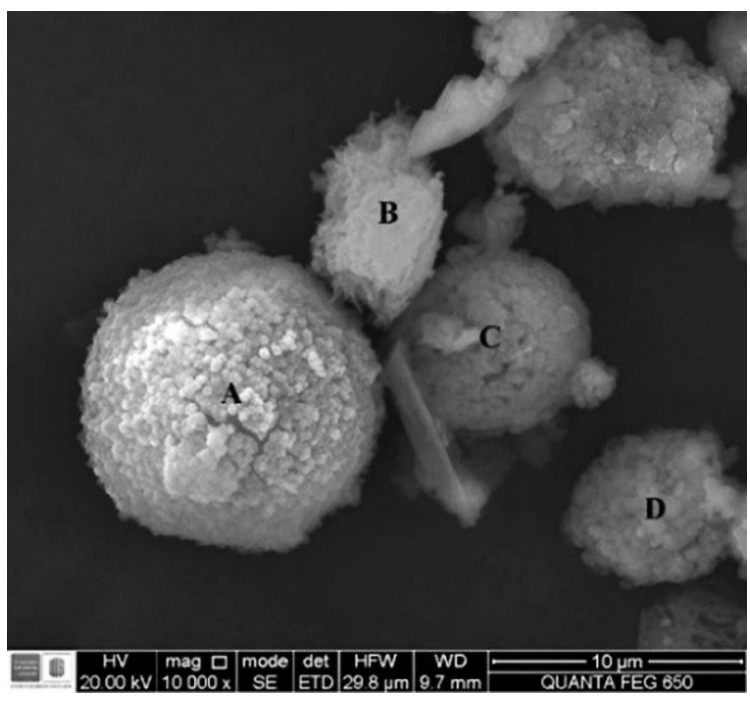

Figura. 10. Microfotografías MEB, Magnificación de 10000X, ZEONa. 
Las ZEONa tienen morfología de acuerdo a la figura 10 de semicírculos bien definidos $\mathrm{A}$ y $\mathrm{C}$, que están agrupados por pequeños sólidos cristalinos, sin embargo, B y D presentan formas desproporcionadas en donde $\mathrm{B}$ se encuentra entre estructuras alargadas, también se aprecia material amorfo que no se estructuró.

Este tipo de zeolitas presentan estructura cristalina de agregados microesféricos de menor tamaño, así mismo cuenta con una morfología semejante a otras investigaciones realizadas [15], [26], [27].

Las muestras ZEOK de la figura 11 se analizaron con una magnificación de 2000X. Se encontró que el promedio de las muestras analizadas es de 4,21 $\mu \mathrm{m}$, con una desviación estándar de 0,41 , valor mínimo de 3,45 $\mu \mathrm{m}$, y un valor máximo de $4,89 \mu \mathrm{m}$.

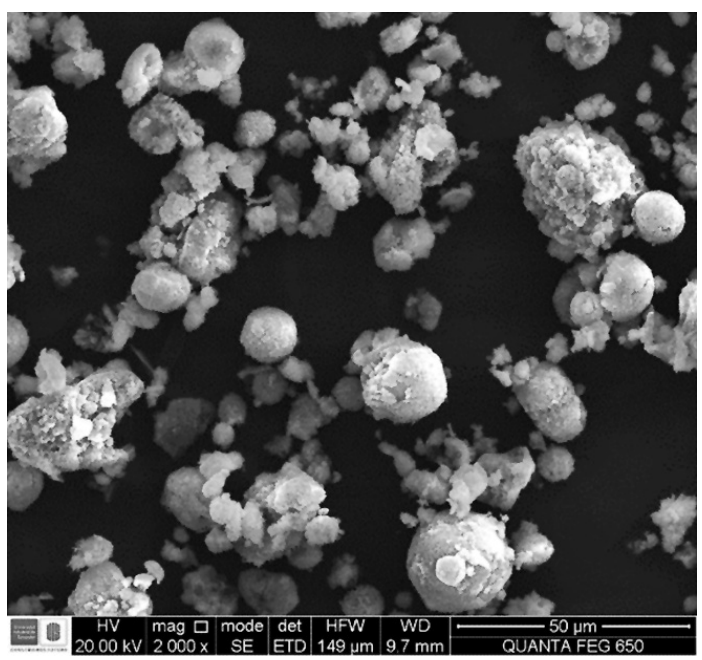

Figura. 11. Microfotografías de la muestra ZEOK, usando MEB FEI Quanta FEG 650.

En la figura 12 se identificó que los diámetros de las partículas A, B, y C son $11,87 \mu \mathrm{m}, 6,56$ $\mu \mathrm{m}, 2,65 \mu \mathrm{m}$ respectivamente. La granulometría estudiada de ZEOK permitió identificar valores de diámetros comprendidos entre 2,65 $\mu \mathrm{m}$ y $11,87 \mu \mathrm{m}$, confirmando lo reportado en otras investigaciones para este tipo de zeolitas [19], [28].

La descripción morfológica de las zeolitas activadas mediante hidrogel en $\mathrm{KOH}$ es semejante a los reportados en los trabajos en Murayama, et al., 2008 y Fukasawa, et al., 2017.

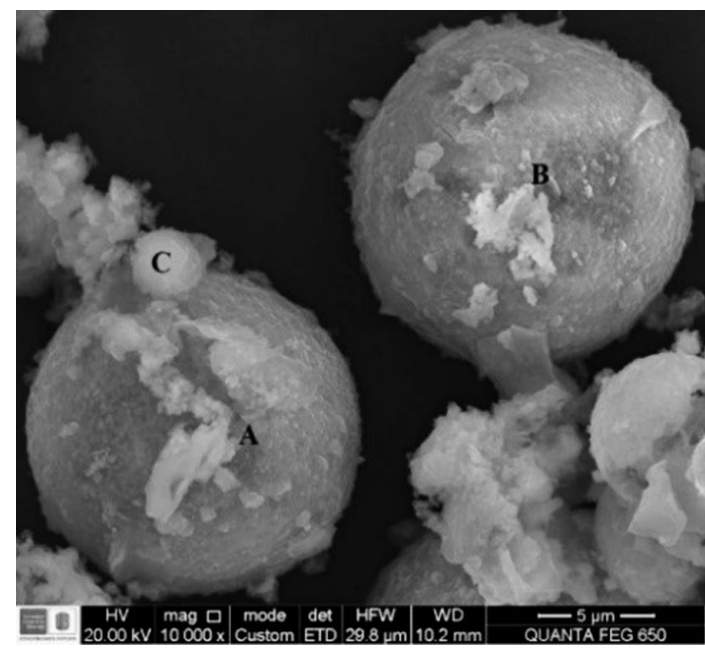

Figura. 12. Microfotografías MEB, Magnificación de 10000X, ZEOK.

Es apreciable formas semiesféricas de superficie fibrosa formados a partir de microcristales que dan lugar al material zeolítico, existen estructuras en formación que al parecer no lograron constituir la semiesfericidad apreciable en $\mathrm{A}, \mathrm{B}, \mathrm{C}$, e igualmente hay acumulación de microcristales en configuraciones irregulares y material amorfo saliente de las partículas.

El estudio cuantitativo de la estructura cristalina de las muestras, se presenta en la tabla 5 y 6, los cuales se obtuvieron por refinamiento Rietveld usando el software XPert HighScore 2.0 a los patrones de difracción de las muestras (ver figura 13). De estos resultados, se observa que el porcentaje de fases cristalina vario de $0,2 \% \mathrm{Wt}$ a $67,5 \% \mathrm{Wt}$ en la muestra ZEONa, y de $0,2 \% \mathrm{Wt}$ a $43,9 \% \mathrm{Wt}$ en la muestra ZEOK. Se aprecia que la muestra ZEONa reporto la fase cristalina de zeolita Natrosilita $\mathrm{Na}_{16.00} \mathrm{Si}_{16.00} \mathrm{O}_{40.00}$. De igual manera se observan la presencia de fases cristalinas de cuarzo, mullita, magnetita y nontronita. En la muestra ZEOK se puede ver la formación de zeolita K-F $\mathrm{K}_{13.5}(\mathrm{OH})_{3} \quad\left(\mathrm{H}_{2} \mathrm{O}\right)_{13}\left(\mathrm{Si}_{10} \mathrm{Al}_{10} \mathrm{O}_{40}\right)$, junto con las fases cristalinas de cuarzo, mullita, carbonato de potasio y oxalato ferroso. Lo anterior se realizó, al comparar los patrones de difracción para cada muestra con los reportados en las bases de datos de PDF-ICDD, a través de las tarjetas PDF No. 96-210-0189, 96-900-1323, 96-9007073, 96-900-6318, 96-901-0768, 98-004-0810, 98010-9188, 98-000-6099, 98-003-6830, 98-0099044. 


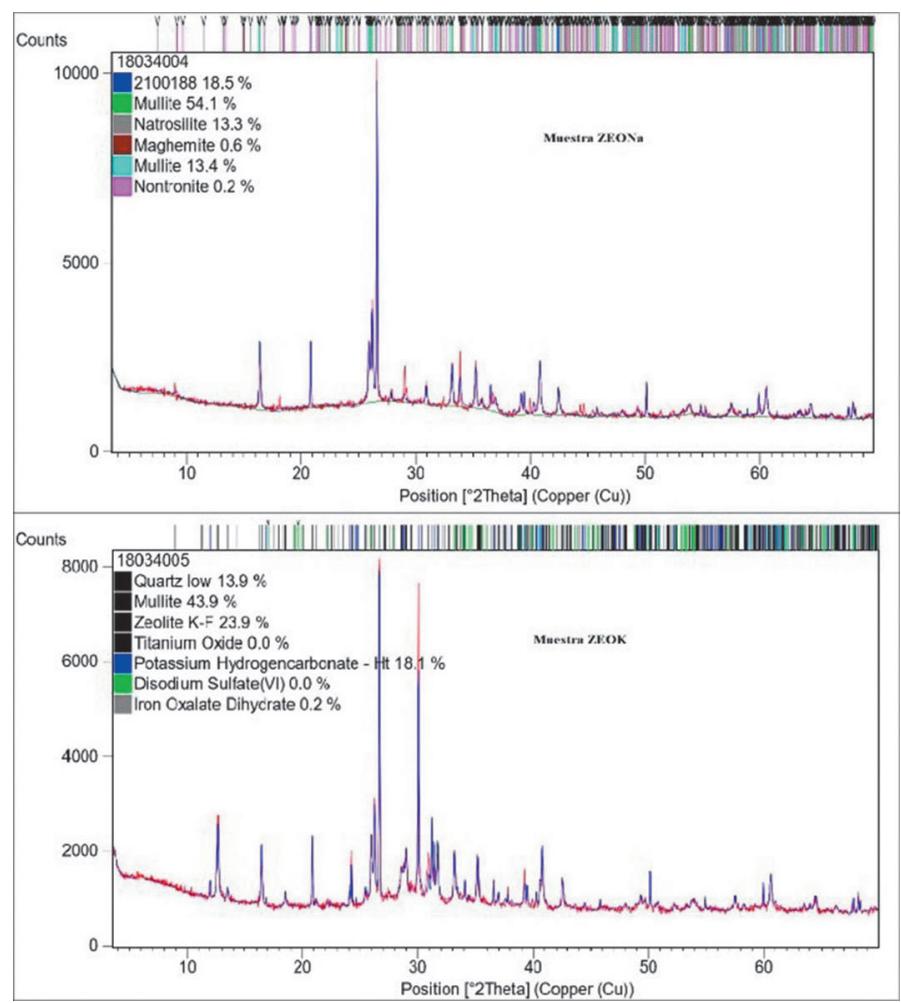

Figura 13. Patrones de DRX y su refinamiento para las muestras ZEONa y ZEOK.

Tabla 5. Resultados difracción de rayos X (DRX) ZEONa. Análisis de concentraciones en peso de las fases cristalinas

\begin{tabular}{cccc}
\hline Fase & Código & Nombre & $\% \mathrm{Wt}$ \\
\hline $\mathrm{Si}_{3.00} \mathrm{O}_{6.00}$ & $96-210-0189$ & Cuarzo & 18,5 \\
$\mathrm{Al}_{4.80} \mathrm{Si}_{1.20} \mathrm{O}_{9.60}$ & $96-900-1323$ & Mullita & 67,5 \\
$\mathrm{Na}_{16.00} \mathrm{Si}_{16.00} \mathrm{O}_{40.00}$ & $96-900-7073$ & Natrosilita & 13,3 \\
$\mathrm{Fe}_{21.33} \mathrm{O}_{32.00}$ & $96-900-6318$ & Magnetita & 0,6 \\
$\mathrm{Na}_{0.82} \mathrm{Fe}_{4.00} \mathrm{Si}_{8.00} \mathrm{O}_{20.02}$ & $96-901-0768$ & Nontronita & 0,2 \\
\hline
\end{tabular}

Tabla 6. Resultados difracción de rayos X (DRX) ZEOK. Análisis de concentraciones en peso de las fases cristalinas

\begin{tabular}{cccc}
\hline Fase & Código & Nombre & $\% \mathrm{Wt}$ \\
\hline $\mathrm{SiO}_{2}$ & $98-004-0810$ & Cuarzo & 13,9 \\
$\left(\mathrm{Al}_{2.34} \mathrm{Si}_{0.66}\right) \mathrm{O}_{4.83}$ & $98010-9188$ & Mullita & 43,9 \\
$\mathrm{~K}_{13.5}(\mathrm{OH})_{3}\left(\mathrm{H}_{2} \mathrm{O}\right)_{13}\left(\mathrm{Si}_{10} \mathrm{Al}_{10} \mathrm{O}_{40}\right)$ & $98-000-6099$ & Zeolita K-F & 23,9 \\
$\mathrm{~K}\left(\mathrm{HCO}_{3}\right)$ & $98-003-6830$ & Carbonato de potasio & 18,1 \\
$\mathrm{Fe}\left(\mathrm{C}_{2} \mathrm{O}_{4}\right)\left(\mathrm{H}_{2} \mathrm{O}\right)_{2}$ & $98-009-9044$ & Oxalato ferrosos & 0,2 \\
\hline
\end{tabular}

El análisis de CIC permitió evaluar la gran capacidad de intercambiar cationes por cada cien gramos de muestra. El valor más alto de
CIC presentado fue el de la muestra de ZEONa obteniendo un valor de $141 \mathrm{meq} / 100 \mathrm{~g}$, los cuales están en el mismo rango que los valores 
de CIC reportados en la literatura [16], en la que se menciona que "las zeolitas tienen una (CIC) entre 100 y $300 \mathrm{meq} / 100 \mathrm{~g}$, la que depende de la cantidad de átomos de aluminio reemplazables en la estructura por átomos de silicio". La muestra de ZEOK, presentó menor capacidad de intercambiar cationes que las ZEONa con valor de CIC de 111,7 meq/g. La estructura del marco zeolítico para la sorción de cationes en ciertas zeolitas es más importante que su relación $\mathrm{Si} /$ Al [31], ya que la estructura por celda unitaria se ve distorsionada por el cambio del número de cationes y tamaño de los mismos, igualmente la selectividad de los cationes $\mathrm{Na}^{+}$y $\mathrm{K}^{+}$depende de la estructura cristalina [32] lo cual justifica la elevada CIC de las ZEONa.

La alta capacidad de intercambiar cationes de las zeolitas sintetizadas en la presente investigación se asocia a la baja relación $\mathrm{Si} / \mathrm{Al}$ [25].

\subsection{Síntesis por el Método de Hidrogel}

El proceso de cristalización de las fases solubles de Si y Al en medio acuoso, permitió la transformación del gel amorfo en zeolitas activadas térmicamente a baja temperatura $90{ }^{\circ} \mathrm{C}$, lo que conlleva a un contenido bajo de silicio en las especies zeolíticas de acuerdo a lo declarado por Gómez, 2001, tal y como lo expresan las composiciones químicas del EDS.

Las fases predominantes de $\mathrm{Si}$ y $\mathrm{Al}$ de las cenizas volantes de Termotasajero S.A.S son fases cristalinas de mullita $\left(\mathrm{Al}_{4.68} \mathrm{Si}_{1.32} \mathrm{O}_{9.66}\right)$ (45\%), seguido del cuarzo $\left(\mathrm{Si}_{3} \mathrm{O}_{6}\right)$ con un $40 \%$, [1], por lo que el radio de disolución será bajo y se reducirá el rendimiento de la zeolitización, pero en la presente investigación se lograron sintetizar zeolitas con bajos contenidos de impurezas, gracias a que se estableció una alta relación entre la solución activante-ceniza volante y periodos de cristalización altos favoreciendo la zeolitización a través de este proceso, según lo reportado en [18]. Por consiguiente, al utilizar el activante $\mathrm{KOH}$ el efecto del $\mathrm{K}^{+}$en la disolución alcalina causa estructuración zeolítica a razón de largos periodos de tiempo [33], lo que confirma la objetividad de mantener fija la variable tiempo por una hora más en las zeolitas activadas con $\mathrm{KOH}$ con respecto a las activadas con $\mathrm{NaOH}$, ya que las fases solubles activadas con $\mathrm{KOH}$ tardarían más tiempo en disolverse por completo, además la eficiencia de conversión a la misma temperatura utilizando soluciones de $\mathrm{KOH}$ y $\mathrm{NaOH}$ requería de tiempos de reacción más prolongados [4].

Se observó que al usar los parámetros establecidos en la metodología de síntesis se obtuvo una buena cristalización de las fases inorgánicas de las cenizas volantes al utilizar agente de álcalis $\mathrm{NaOH}$ y $\mathrm{KOH}$, síntesis que se ve influenciada por la solución activante, lo que permitió producir zeolitas natrosilita y zeolitas K-F.

Las zeolitas sintetizadas poseen estructura de microcristales del tipo natrosilita y zeolita K-F, de acuerdo a ello estas pueden emplearse en aplicaciones industriales, tales como:

Zeolita Natrosilita: Se puede adaptar y aplicar en súper-hidratación y expansión de volumen inducida por presión [34]. Pueden ajustarse como tamices moleculares de intercambio iónico y de catálisis [35]. Proporciona aplicaciones e investigaciones novedosas sobre la modificación de la geometría y la química de los nanoporos en los canales estructurales de intercambio de cationes [36]. Se ha usado como catalizador reutilizable y como instrumento selectivo para aislar hidrógeno o helio [37].

Zeolita K-F: útiles en los procesos químicos industriales que involucran procesos catalíticos y de adsorción debido a sus características hidrofílicas y básicas [38]. Este tipo de zeolita puede aplicarse también como tamiz molecular, en la adsorción e intercambio iónico [39]. Alta selectividad de $\mathrm{NH}_{4}^{+}$, en la limpieza de aguas residuales [40]. El cristal de zeolita K-F, tiene una alta capacidad de intercambio catiónico, y podría ser utilizado para la enmienda del suelo para eliminar otros cationes [41].

\section{CONCLUSIONES}

La composición química por elemento usando (EDS) demostró que los elementos principales de las zeolitas son $\mathrm{Si}, \mathrm{Al}, \mathrm{O}, \mathrm{Na}, \mathrm{K}, \mathrm{Ca}, \mathrm{Fe}, \mathrm{y}$ en proporciones menores $\mathrm{Ti}$ y $\mathrm{Mg}$. Las ZEONa presentaron relación Si/Al igual a 1,19 y las ZEOK valor de 1,00 , estas zeolitas se clasifican 
como zeolitas de baja relación $\mathrm{Si} / \mathrm{Al}$, con carácter hidrófilo. Lo anterior es debido a la presencia de hierro en las CV, dificultando la disolución del Si y Al durante el proceso de cristalización, ya que el óxido de hierro se distribuye en la superficie de las partículas $\mathrm{CV}$.

Los resultados obtenidos en el análisis morfológico mediante (MEB) de las zeolitas permitieron identificar que las ZEONa tienen diámetros de partícula que varían en un rango de 4,94 $\mu \mathrm{m}$ y 11,66 $\mu$ mcon formas semiesféricas agrupadas por pequeños sólidos cristalinos, y las ZEOK tienen partículas con valores entre $2,65 \mu \mathrm{my}$ $11,87 \mu \mathrm{m}$ en los que se observan conglomerados de micropartículas semiesferoidal, así como también se evidencia mayor cantidad de fracciones de material zeolítico en proceso de zeolitización. Así mismo en las microfotografías para las ZEONa y ZEOK se percibe la existencia de material amorfo en formas alargadas entrecruzadas.

La caracterización del producto zeolítico con respecto a la prueba de intercambio catiónico permitió establecer una mayor capacidad de intercambio catiónico de las ZEONa con respecto a las ZEOK, carácter que se atribuye a la baja relación $\mathrm{Si} / \mathrm{Al}$ asociado a los iones intercambiables de $\mathrm{Na}^{+}$. Esta propiedad de una elevada capacidad de intercambiar cationes les confiere a las zeolitas la facultad como agentes deshidratantes y adsorbentes.

Se evidenció que es posible sintetizar zeolitas a partir de cenizas volantes en gel de reacción con disoluciones de $\mathrm{NaOH}$ y $\mathrm{KOH}$. De acuerdo a los resultados reportados de rayos $\mathrm{X}$ se logró producir zeolitas del tipo natrosilita y zeolita K-F en condiciones de baja temperatura a través del método de hidrogel.

\section{AGRADECIMIENTOS}

Al Grupo de Investigación en Instrumentación y Física de la Materia Condensada (GIFIMAC) de la Universidad Francisco de Paula Santander (UFPS), a través del cual se efectuó el desarrollo de la investigación.

\section{REFERENCIAS}

[1] Peña, G., y Ortega, L, "Caracterización Morfológica y Estructural de Polvos de Cenizas Volantes" [online]. $I^{2}+D, 14(2)$, 14-19, 2014. Disponible en: Dialnet-Carac terizacionMorfologicaYEstructuralDePolv osDeCe-6096183.pdf.

[2] Centro de Estudios y Experimentación de Obras Públicas, "Cenizas volantes de carbón y cenizas de hogar o escorias (3.1)" [online]. 2011. Disponible en: http://www.cedex.es/NR/rdonlyres/ B 01FDCCB-AC 8E-4089-9699-FA $6413 \mathrm{~F} \mathrm{~B} \mathrm{E} \mathrm{E} 7 \mathrm{C} / 1119905 /$ C E N I Z A S V O L A N T E S DECARBONYCENIZASDEHOGAR.pdf.

[3] Calleja, A, "La Importancia de las Zeolitas" [online]. Revista cuadernos tomas, CT (1), 211-227, 2009. Disponible en: https://dialnet.unirioja.es/servlet/ articulo? codigo $=3760692$.

[4] Farro, N., Reyes, W., Díaz, J., Mendoza, J., Chuquimango, B., y Araujo, C, "Influencia de la proporción de hidróxido de sodio a cenizas volátiles en la obtención de zeolitas para purificar efluentes acuosos contaminados con metales pesados" [online]. Revista Ciencia y Tecnología, (3), 127-140, 2015. Disponible en: http:// revistas.unitru.edu.pe/index.php/PGM/ article/view/1120/1046.

[5] Gómez, J. Síntesis, caracterización y aplicaciones catalíticas de zeolitas básicas. Universidad Complutense de Madrid. Madrid, España, 2001.

[6] Murayama, N., Yamamoto, H., y Shibata, $\mathrm{J}$, "Zeolite synthesis from coal fly ash by hydrothermal reaction using various alkali sources ${ }^{+}$. Journal of chemical Technology and Biotechnology, 77, 280-286, 2002. Doi: 10.1002/jctb. 604 .

[6] González, D., Pérez, L., Santa, A., y Ramírez, J, "Producción y caracterización de zeolita obtenida a partir de ceniza volante mediante drx" [online]. MOMENTO 
Revista de Física, (48), 52-60, 2014. Disponible en: http://www.bdigital.unal. edu.co/44963/1/45542-219568-2-PB.pdf.

[7] Hernández, J., González, J., Carruyo, G., y García, C, "Lixiviación diferenciada de metales mayoritarios de cenizas volantes" [online]. Revista Tecnocientifica URU, (9), 19-31, 2015. Disponible en: http://200.35.84.134/ojs-2.4.2/index.php/ rtcu/article/viewFile/294/pdf_17.

[8] Chica F., Londoño, L., y Álvarez, M, "La zeolita en la mitigación ambiental" [online]. Revista Lasallista de Investigación, 3(1), 2006. Disponible en: http://www.redalyc. org/articulo.oa? $\mathrm{id}=69530106$.

[9] Guía Técnica Colombiana GTC, 35, 1997.

[10] Martínez, D., \& Cicuamía A, Síntesis y caracterización de zeolitas a partir de cenizas volantes de carbón. Universidad de Ciencias Aplicadas y Ambientales. Bogotá, Colombia, 2016.

[11] Norma ASTM: C 117-03 Standard Test Method for Materials Finer than 75- $\mu \mathrm{m}$ (No. 200) Sieve in Mineral Aggregates by Washing.

[12] Velásquez, L.F., De la Cruz, J.F., Sánchez, J.F., y Marín, M.A, "Remoción de Carbón Inquemado de las Cenizas Volantes Producidas en el Proceso de Combustión de Carbón" [online]. Revista Energética, (38), 107-112, 2007. Disponible en: https:// revistas.unal.edu.co/index.php/energetica/ article/viewFile/9508/10105.

[13] Saavedra, M.L., Cuevas, D., Saldivia, M., y Santoro, R, "Remoción de iones Cromo y Cobre desde soluciones acuosas con cenizas volantes de Carbón mineral" [online]. Revista de la Facultad de Ciencias Químicas, (12), 10-19, 2015. Disponible en: $\quad$ http://dspace.ucuenca.edu.ec/ bitstream/123456789/23871/1/2_articulo_ revista_12.pdf.
[14] Harja, M., Bucur, D., Cimpeanu, S.M., Ciocinta, R.C., y Gurita, A.A, "Conversion of ash on zeolites for soil application" [online]. WFL Publisher Science and Technology, 10(2), 1056-1059, 2012. Disponible en: https://www.researchgate. net/publication/227339645_Conversion_ of_ash_on_zeolites_for_soil_application.

[15] Shoumkova, A., Stoyanova, V., Tsacheva, T., y Avdeev, G, "Comparative Study on the Zeolitization of Coal Fly Ash from TPP "Maritsa 3" with $\mathrm{KOH}$ and $\mathrm{NaOH}$ " [online]. Ecology and Environmental Protection, 159-166, 2016. Disponible en: https://www. researchgate.net/publication/268402487.

[16] Jiménez, M, J, Caracterización de Minerales Zeolíticos mexicanos. Universidad Autónoma del Estado de México. Toluca, México, 2004.

[17] Bhagwanjee, J., Padmakumar, G., Singh, D., y Kannan, I, "Synthesis of zeolites by fly ash alkali interaction" [online]. Indian Geotechnical Conference, Kochi (R-243), 1089-1092, 2001. Disponible en: https:// gndec.ac.in/ igs/ldh/conf/2011/articles/ Theme\%20-\%20R\%205.pdf.

[18] Benavides, P., \& Mendoza, E, Síntesis de zeolitas a partir de cenizas de carbón provenientes de la combustión de la termoeléctrica de Zipaquirá. Universidad Industrial de Santander. Bucaramanga, Colombia, 2007.

[19] Martínez, J, Obtención de Zeolitas Utilizando Líquidos Iónicos como Agentes Directores de Estructura. Universidad de Sevilla. Sevilla, España, 2015.

[20] Yuncosa, R, Síntesis y Caracterización de Zeolita y Mediante Calentamiento por Microondas. Universidad Central de Venezuela. Caracas, Venezuela, 2008.

[21] Jha, B., y Narain, D. (Eds), "Fly Ash Zeolites". 2016. doi: 10.1007/978-981-101404-8. 
[22] Arroyave, J.C., Arboleda, J.C., Hoyos, D.A., y Echavarría, A.P, "LTA and FAU zeolites from coal combustion and residue by products for Chromium removal application". DYNA, 85(204), 150-160, 2017. http://dx.doi.org/10.15446/dyna. v85n204.67096.

[23] Hai, S., Hu Sik, K., Sung, M.S., Seong, O.K., Jeong, M.S., Ghyung, H.K., y Woo, T.L, "Location of $\mathrm{Na}+$ Ions in Fully Dehydrated $\mathrm{Na}^{+}$-saturated Zeolite $\mathrm{Y}$ (FAU, Si/Al = 1.56)" [online], Bull Korean Chem Soc, 33(8), 2785-2788, 2012. Disponible en: http://koreascience. or.kr/article/Article FullRecord. jsp?cn=JCGMCS_2012_v33n8_2785.

[24] Flores, C.G., Schneider, H., Marcilio, N.R., Ferret, L., y Pinto, J.C, "Potassic zeolites from Brazilian coal ash for use as a fertilizer in agricultura". ELSEIVER, (2017), 1-9, 2017. http://dx.doi.org/10.1016/j. wasman.2017.08.039.

[25] Mosqueda, D.B, "Estudios de Zeolitas Naturales y Modificadas para su Utilización como Catalizadores de Control AmbientalEdicion Única”. Instituto Tecnológico y de Estudios Superiores de Monterrey. Monterrey, México, 1997.

[26] Kunecki, P., Panek, R., Wdowin. M., y Franus, W, "Synthesis of faujasite (FAU) and tschernichite (LTA) type zeolites as a potential direction of the development of lime class C fly ash". International Journal of Mineral Processing, 2017. http://dx.doi. org/10.1016/j.minpro.2017.07.007.

[27] Chen, J., Ma, H., Liu, C., y Yuan J, "Synthesis of Analcime Crystals and Simultaneous Potassium Extraction from Natrolite Syenite". Hindawi, 2017, 1-9, 2017. https:// doi.org/10.1155/2017/2617597.

[28] Barbosa, A., Barbosa, A., Santos, E., Leite, R., Rodrigues, M, “Obtención de la Zeolitas MCM-22 a través de la Síntesis Hidrotermal Utilizando Diferentes Métodos" [online]. Avances en Ciencias e Ingenieria, 3(3), 59-67, 2012. Disponible en: http://www. redalyc.org/pdf/3236/323627687007.pdf
[29] Murayama, N., Takahashi, T., Shuku., K., Lee, H., y Shibata, J, "Effect of reaction temperature on hydrothermal Syntheses of potassium type zeolites from coal fly ash". Journal of Mineral Processing, 87(2008), 129-133, 2008. doi:10.1016/j. minpro.2008.03.001.

[30] Fukasawa, T., Horigome, A., Tsu, T., Karisma, A.D., Maeda, N., Huang, A., y Fukui, K, "Utilization of incineration fly ash biomass power plants for zeolite Synthesis from coal fly ash hydrothermal treatment". Fuel Processing Technology, 167(2017), 92-98, 2017. http://dx.doi.org/10.1016/j. fuproc.2017.06.023.

[31] Baek, W., Ha, S., Hong, S., Kim S., y Kim, $\mathrm{Y}$, "Cation exchange of cesium and cation selectivity of natural zeolites: Chabazite, stilbite, and heulandite". ELSEIVER, 624(2018), 159-166, 2018. https://doi. org/10.1016/j.micromeso.2018.01.025.

[32] Auerbach, S.M., Carrado, K.A., y Dutta, P.K, "Handbook of Zeolite Science and Technology”. New York, Estados Unidos. Editorial Taylor \& Francis Group, 2003.

[33] Arango, N., Pérez, J., Gochi, Y., y Sánchez, M, "Zeolitas a partir de cenizas volantes generadas en las centrales carboeléctricas para el tratamiento de agua contaminada con Mn y Pb" [online]. Tecnología, Ciencia, Educación, 26(1), 5-13, 2011. Disponible en: http://www.redalyc.org/articulo. oa? id=48219771002.

[34] Yongjaa, L., Thomas, V., Hriljac, J., Parise J., y Artiori G, "Pressure-Induced Volume Expansion of Zeolites in the Natrolite Family". American Chemical Society 124(2002), 5466-5475, 2002. 10.1021/ ja0255960

[35] Grima, J., Gatt, R., Zammit, V., Williams, J., y Evans, K, "Natrolite: A zeolite with negative Poisson's ratios". Journal of Applied Physics 101(086102), 2007. http:// dx.doi.org/10.1063/1.271889. 
[36] Seryotkin, Y., Bakakin, V., Likhacheva, A., Dementiev, S., y Rashchenko, S., "Structural behavior of Tl-exchanged natrolite at high pressure depending on the composition of pressure-transmitting medium". Phys Chem Minerals, 2017. DOI 10.1007/s00269-017-0887-0.

[37] Li, H., Zhou, X., Zhang, J., Wu, X., y Gao, H., "The Preparation and Characterization of Natrolite Synthetized by Purified Attapulgite". The 2nd International Conference on Materials Engineering and Nanotechnology, 205(012030), 2017. 10.1088/1757-899X/205/1/012030

[38] Wong, S., Awala, H., Vincente, A., Retoux, R., Ling, T., Mintova, S, Mukti, R., y Ng, E., "K-F Zeolite Nanocrystals Synthesized From Organic-template-free Precursor Mixture". Microporous and Mesoporous Materials, 17(1811), 2017. 10.1016j. micromeso.2017.04.053
[39] Wong, S., Wittayakun, J., Ling, T., Muraza, O., Adam, F., y Ng, E., "Crystal Growth Study of K-F Nanozeolite and Its Catalytic Behavior in Aldol Condensation of Benzaldehyde and Heptanal". Materials Chemistry and Physics, 17(0584), 2017. 10.1016/j.matchemphys.2017.04.061

[40] Novembre, D., Pace, C., y Gimeno, D., "Syntheses and characterization of zeolites K-F and W type using a diatomite precursor". Mineralogical Magazine, 78(5), 1209-1225, 2014. 10.1180/minmag.2014.078.5.08

[41] Wajima, T., y Munakata, K., "Effect of alkali species on synthesis of K-F zeolitic materials from paper sludge ash for soil amendment". Chemical Engineering Journal 207(208), 906-912, 2012. http:// dx.doi.org/10.1016/j.cej.2012.06.136 